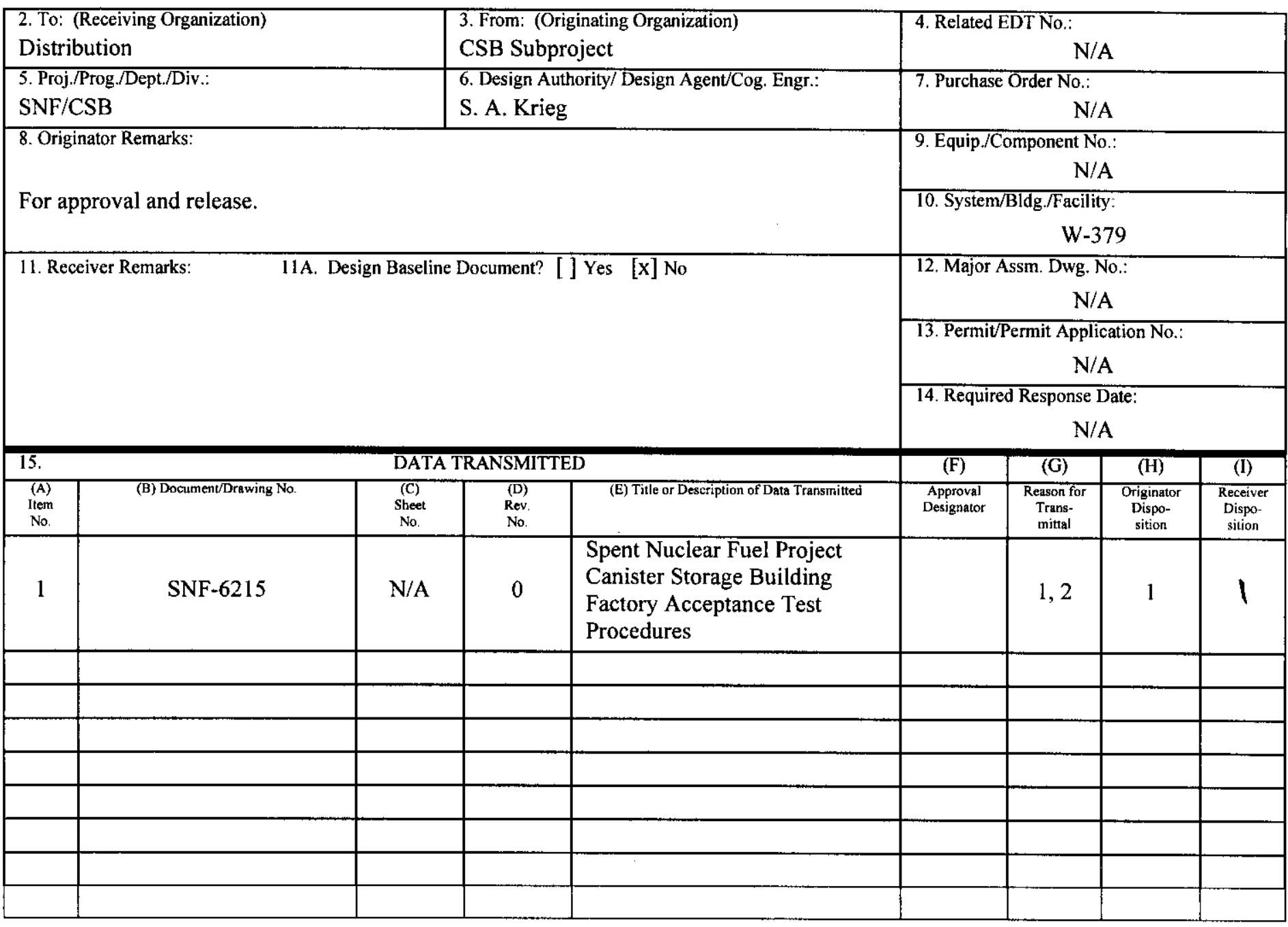

\begin{tabular}{|c|c|c|c|c|c|c|c|}
\hline \multicolumn{8}{|c|}{ KEY } \\
\hline \multicolumn{3}{|c|}{ Approval Designator (F) } & \multicolumn{3}{|c|}{ Reason for Transmittal (G) } & \multicolumn{2}{|c|}{ Disposition $(\mathrm{H}) \&(\mathrm{l})$} \\
\hline \multicolumn{3}{|c|}{$\begin{array}{l}\text { E, S, Q, D or N/A } \\
\text { (see WHC-CM-3-5, Sec.12.7) }\end{array}$} & $\begin{array}{l}\text { 1. Approval } \\
\text { 2. Release } \\
\text { 3. Information }\end{array}$ & \multicolumn{2}{|l|}{$\begin{array}{l}\text { 4. Review } \\
\text { 5. Post-Review } \\
\text { 6. Dist. (Receipt Acknow. Required) }\end{array}$} & \multicolumn{2}{|c|}{$\begin{array}{ll}\text { 1. Approved } & \text { 4. Reviewed no/comment } \\
\text { 2. Approved w/comment } & \text { 5. Reviewed w/comment } \\
\text { 3. Disapproved w/comment } & \text { 6. Receipt acknowledged }\end{array}$} \\
\hline \multicolumn{8}{|c|}{$\begin{array}{c}\text { 17. SIGNATURE/DISTRIBUTION } \\
\text { (See Approval Designator for required signatures) }\end{array}$} \\
\hline $\begin{array}{c}\text { (G) } \\
\text { Reason }\end{array}$ & $\begin{array}{l}\text { (H) } \\
\text { Disp. }\end{array}$ & \multicolumn{3}{|c|}{ (J) Name $\quad$ (K) Signature (L) Date (M) MSIN } & $\begin{array}{c}\text { (G) } \\
\text { Reason }\end{array}$ & $\begin{array}{c}\text { (H) } \\
\text { Disp. }\end{array}$ & (J) Name \\
\hline \multirow[t]{3}{*}{1} & 1 & \multicolumn{3}{|c|}{ Design Authority S. A. Krieg 4 U Reg 4-19-00 } & 1 & 1 & Startup R. J. Jablonski \\
\hline & & \multicolumn{3}{|c|}{ Design Agent N/A } & 1 & 1 & Construction IHAmertime \\
\hline & & \multicolumn{3}{|c|}{ Cog. Eng. N/A } & & & J.T.Koberg \\
\hline \multirow[t]{4}{*}{1} & / & \multicolumn{2}{|c|}{ Cog. Mgr. G. } & 10 & & & \\
\hline & & \multicolumn{2}{|c|}{ QA N/A } & Z & & & \\
\hline & & \multicolumn{2}{|c|}{ Safety N/A } & & & & \\
\hline & & \multicolumn{3}{|c|}{ Env. N/A } & & & \\
\hline
\end{tabular}
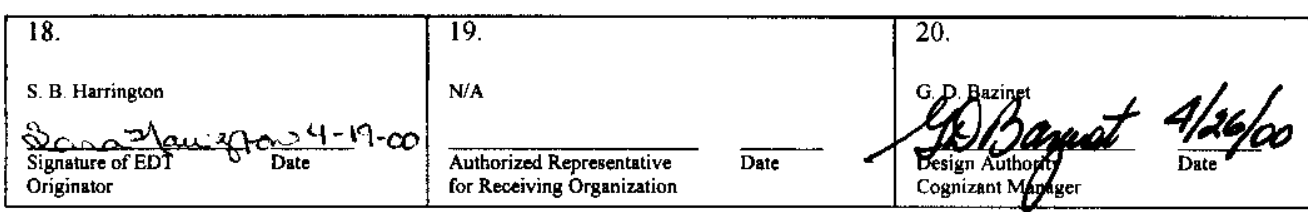

21. DOE APPROVAL (if required)

Ctrl. No.

[] Approved

[ ] Approved w/comments

[] Disapproved w/comments 


\section{Spent Nuclear Fuel Project Canister Storage Building Factory Acceptance Test Procedures}

Prepared for the U.S. Department of Energy

Assistant Secretary for Environmental Management

Project Hanford Management Contractor for the

U.S. Department of Energy under Contract DE-AC06-96RL13200

Fluor Hanford

P.o. Box 1000

Richland, Washington 


\section{Spent Nuclear Fuel Project Canister Storage Building Factory Acceptance Test Procedures}

G. D. Bazinet

Total Pages: 17

Fluor Hanford

Date Published

April 2000

Prepared for the U.S. Department of Energy

Assistant Secretary for Environmental Management

Project Hanford Management Contractor for the

U.S. Department of Energy under Contract DE-AC06-96RL13200

Fluor Hanford

P.O. Box 1000

Richland, Washington
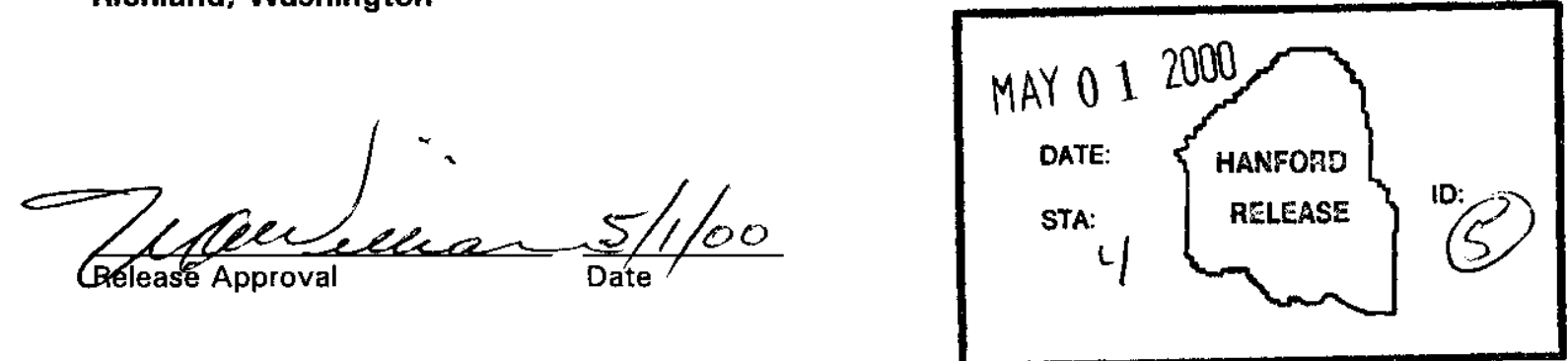
TRADEMARK DISCLAIMER

Reference herein to any specific commercial product, process, or service by trade name, trademark, manufacturer, or otherwise, does not necessarily constitute or imply its endorsement, recommendation, or favoring by the United States Government or any agency thereof or its contractors or subcontractors.

This report has been reproduced from the best available copy.

Printed in the United States of America 


\section{Spent Nuclear Fuel Project Canister Storage Building Factory Acceptance Test Procedures}

\begin{tabular}{|c|c|c|c|c|}
\hline & Section & Para & Submittal & DESGRIPTION \\
\hline & C-08361 & & & RADIATION SHIELD DOORS \\
\hline 1 & C-08361 & 2.3.1 & 0 & Factory Acceptance Test Procedures (FAT) - Overly Manufacturing Company \\
\hline 2 & C-08361 & 2.3 .1 & 1 & Factory Acceptance Test Procedures (FAT) - Overly Manufacturing Company \\
\hline & P-13088 & & & CANISTER/OVERPACK-STORAGE TUBE ASSEMBLIES \\
\hline 3 & P-13088 & 1.6 .2 & 0 & $\begin{array}{l}\text { Procedures for Fabrication, Inspection, Repair, Factory Acceptance Tests (FATs), } \\
\text { Handling, Cleaning, Packaging - American Boa }\end{array}$ \\
\hline 4 & P-13088 & 1.6.2.D & 0 & $\begin{array}{l}\text { Factory Acceptance Test Procedure (FAT) - Pneumatic Pressure Test, No PP-015 - } \\
\text { Oregon Iron Works }\end{array}$ \\
\hline 5 & P-13088 & 1.6.2.D & $\mathrm{OA}$ & $\begin{array}{l}\text { Factory Acceptance Test Procedure (FAT) - Pneumatic Pressure Test, No PP-015, Rev } \\
1 \text { - Oregon Iron Works }\end{array}$ \\
\hline 6 & P-13088 & 1.6.2.D & $\mathrm{OB}$ & $\begin{array}{l}\text { Factory Acceptance Test Procedure (FAT) - Pneumatic Pressure Test, No PP-015, Rev } \\
2 \text { - Oregon Iron Works }\end{array}$ \\
\hline 7 & P-13088 & 1.6.2.D & $\mathrm{OC}$ & $\begin{array}{l}\text { Factory Acceptance Test Procedure (FAT) - Pneumatic Pressure Test, No PP-015, Rev } \\
3 \text { - Oregon Iron Works }\end{array}$ \\
\hline 8 & P-13088 & 1.6.2.D & 1 & $\begin{array}{l}\text { Factory Acceptance Test Procedure (FAT) - First Article Test, No PP-016 - Oregon Iron } \\
\text { Works }\end{array}$ \\
\hline 9 & $\mathrm{P}-13088$ & 1.6.2.D & $1 \mathrm{~A}$ & $\begin{array}{l}\text { Factory Acceptance Test Procedure (FAT) - First Article Test, No PP-016, Rev } 1 \text { - } \\
\text { Oregon Iron Works }\end{array}$ \\
\hline 10 & $P-13088$ & 1.6.2.D & 1B & $\begin{array}{l}\text { Factory Acceptance Test Procedure (FAT) - First Article Test, No PP-016, Rev } 2 \text { - } \\
\text { Oregon Iron Works }\end{array}$ \\
\hline 11 & P-13088 & 1.6.2.D & 10 & $\begin{array}{l}\text { Factory Acceptance Test Procedure (FAT) - First Article Test, No PP-016, Rev } 3 \text { - } \\
\text { Oregon Iron Works }\end{array}$ \\
\hline 12 & P-13088 & 1.6.2.D & $1 \mathrm{D}$ & $\begin{array}{l}\text { Factory Acceptance Test Procedure (FAT) - First Article Test, No PP-016, Rev } 4 \text { - } \\
\text { Oregon Iron Works }\end{array}$ \\
\hline 13 & $\mathrm{P}-13088$ & 1.6.3.B & 0 & $\begin{array}{l}\text { Factory Acceptance Test Procedures (FAT) on Tube Sleeves - ST-1-001, ST-1-003, ST- } \\
\text { 1-012, ST-1-024 \& ST-1-088 - Oregon Iron Works (Ref P-05064/1.6.4/8 \& } \\
\text { 13088/1.6.3.A/4) }\end{array}$ \\
\hline 14 & P-13088 & 1.6.3.B & 1 & $\begin{array}{l}\text { Factory Acceptance Test Procedures (FAT) on Tube Sleeves - OlW Shipment 462-04 - } \\
\text { ST- } 1-002, \text { ST-1-009, ST-1-010, ST-1-025 \& Spacer Rings } 1 \text { A - 9, 10, } 11 \text { \& } 12 \text { - Oregon } \\
\text { Iron Works (Ref P-05064/1.6.4/7 \& 13088/1.6.3.A/3) }\end{array}$ \\
\hline 15 & P-13088 & 1.6.3.B & 2 & $\begin{array}{l}\text { Factory Acceptance Test Procedures (FAT) on Tube Sleeves - OIW Shipment } 462-06 \text { - } \\
\text { ST-1-011, ST-1-013, ST-1-022, ST-1-028 \& ST-1-041 - Oregon Iron Works (Ref P- } \\
\text { 05064/1.6.4/9 \& 13088/1.6.3.A/5) }\end{array}$ \\
\hline 16 & P-13088 & 1.6.3.B & 3 & $\begin{array}{l}\text { Factory Acceptance Test Procedures (FAT) on Tube Sleeves - OlW Shipment 462-07 - } \\
\text { ST-1-074, ST-1-075, ST-1-076, ST-1-084 \& ST-1-107 - Oregon Iron Works (Ref P- } \\
\text { 05064/1.6.4/10 \& 13088/1.6.3.A6) }\end{array}$ \\
\hline 17 & P-13088 & 1.6.3.B & 4 & $\begin{array}{l}\text { Factory Acceptance Test Procedures (FAT) on Tube Sleeves - OIW Shipment 462-10- } \\
\text { ST-1-056, ST-1-077, ST-1-128, ST-1-143 \& ST-1-142 - Oregon tron Works (Ref P- } \\
\text { 05064/1.6.4/11 \& 13088/1.6.3.A/7) }\end{array}$ \\
\hline
\end{tabular}




\begin{tabular}{|c|c|c|c|c|}
\hline & Section & Para & Subntutal & DESORIPTION \\
\hline 18 & P-13088 & 1.6.3.B & 5 & $\begin{array}{l}\text { Factory Acceptance Test Procedures (FAT) on Tube Sleeves - OIW Shipment } 462-11 \text { - } \\
\text { ST-1-004, ST-1-097, ST-1-127, ST-1-139 \& ST-1-144 - Oregon Iron Works (Ref P- } \\
\text { 05064/1.6.4/12 \& 13088/1.6.3.A/8) }\end{array}$ \\
\hline 19 & $P-13088$ & $1.6 .3 . \mathrm{B}$ & 6 & $\begin{array}{l}\text { Factory Acceptance Test Procedures (FAT) on Tube Sleeves - OlW Shipment 462-17 - } \\
\text { ST-1-048, ST-1-060, ST-1-063, ST-1-151 \& ST-1-167 - Oregon Iron Works }\end{array}$ \\
\hline 20 & $P-13088$ & $1.6 .3 . \mathrm{B}$ & 7 & $\begin{array}{l}\text { Factory Acceptance Test Procedures (FAT) on Tube Sleeves - OIW Shipment 462-16 - } \\
\text { ST-1-006, ST-1-053, ST-1-064, ST-1-086 \& ST-1-112 - Oregon Iron Works }\end{array}$ \\
\hline 21 & $P-13088$ & 1.6.3.B & 8 & $\begin{array}{l}\text { Factory Acceptance Test Procedures (FAT) on Tube Sleeves - OIW Shipment 462-17 - } \\
\text { ST-1-005, ST-1-032, ST-1-061, ST-1-079 \& ST-1-120 - Oregon Iron Works }\end{array}$ \\
\hline 22 & $P-13088$ & 1.6.3.B & 9 & $\begin{array}{l}\text { Factory Acceptance Test Procedures (FAT) on Tube Sleeves - OIW Shipment 462-08 - } \\
\text { ST-1-017, ST-1-055, ST-1-062, ST-1-071 \& ST-1-083 - Oregon Iron Works (Ref P- } \\
\text { 05064/1.6.4/16 \& 13088/1.6.3.A/12) }\end{array}$ \\
\hline 23 & $P-13088$ & 1.6.3.B & 10 & $\begin{array}{l}\text { Factory Acceptance Test Procedures (FAT) on Tube Sleeves - OIW Shipment 462-09 - } \\
\text { ST-1-018, ST-1-038, ST-1-096, ST-1-110 \& ST-1-126 - Oregon Iron Works (Ref P- } \\
\text { O5064/1.6.4/17 \& 13088/1.6.3.A/13) }\end{array}$ \\
\hline 24 & $P-13088$ & 1.6.3.B & 11 & $\begin{array}{l}\text { Factory Acceptance Test Procedures (FAT) on Tube Sleeves - OIW Shipment 462-12 - } \\
\text { ST-1-008, ST-1-057, ST-1-091, ST-1-104 \& ST-1-145 - Oregon Iron Works (Ref P- } \\
\text { 05064/1.6.4/18 \& 13088/1.6.3.A14) }\end{array}$ \\
\hline 25 & P-13088 & 1.6.3.B & 12 & $\begin{array}{l}\text { Factory Acceptance Test Procedures (FAT) on Tube Sleeves - OIW Shipment 462-13- } \\
\text { ST-1-040, ST-1-051, ST-1-069, ST-1-115 \& ST-1-184 - Oregon Iron Works (Ref P- } \\
\text { 06064/1.6.4/19 \& 13088/1.6.3.A15) }\end{array}$ \\
\hline 26 & $P-13088$ & 1.6.3.B & 13 & $\begin{array}{l}\text { Factory Acceptance Test Procedures (FAT) on Tube Sleeves - OIW Shipment } 462-19 \text { - } \\
\text { ST-1-027, ST-1-044, ST-1-108, ST-1-117 \& ST-1-173 - Oregon Iron Works (Ref P- } \\
\text { 05064/1.6.4/20 \& 13088/1.6.3.A/16) }\end{array}$ \\
\hline 27 & $\mathrm{P}-13088$ & 1.6.3.B & 14 & $\begin{array}{l}\text { Factory Acceptance Test Procedures (FAT) on Tube Sleeves - OIW Shipment 462-21 - } \\
\text { ST-1-118, ST-1-134, ST-1-135, ST-1-138 \& ST-1-171 - Oregon Iron Works (Ref P- } \\
\text { 05064/1.6.4/21 \& 13088/1.6.3.A/7) }\end{array}$ \\
\hline 28 & P-13088 & 1.6.3.B & 15 & $\begin{array}{l}\text { Factory Acceptance Test Procedures (FAT) on Tube Sleeves - OIW Shipment 462-22 - } \\
\text { ST-1-103, ST-1-121, ST-1-125, ST-1-129 \& ST-1-137 - Oregon Iron Works (Ref P- } \\
\text { 05064/1.6.4/22 \& 13088/1.6.3.A/8) }\end{array}$ \\
\hline 29 & $P-13088$ & 1.6.3.B & 16 & $\begin{array}{l}\text { Factory Acceptance Test Procedures (FAT) on Tube Sleeves - OIW Shipment } 462-23 \text { - } \\
\text { ST-1-023, ST-1-140, ST-1-141, ST-1-168 \& ST-1-217 - Oregon Iron Works (Ref P. } \\
\text { 05064/1.6.4/23 \& 13088/1.6.3.A/19) }\end{array}$ \\
\hline 30 & $P-13088$ & $1.6 .3 . \mathrm{B}$ & 17 & $\begin{array}{l}\text { Factory Acceptance Test Procedures (FAT) on Tube Sleeves - OlW Shipment 462-29 - } \\
\text { ST-1-132, ST- } 1-149, \text { ST-1-189, ST-1-212 \& ST-1-219 - Oregon Iron Works (Ref P- } \\
\text { O5064/1,6,4/24 \& 13088/1.6.3.A/20) }\end{array}$ \\
\hline 31 & P-13088 & 1.6.3.B & 18 & $\begin{array}{l}\text { Factory Acceptance Test Procedures (FAT) on Tube Sleeves - OIW Shipment } 462-30 \text { - } \\
\text { ST-1-124, ST-1-164, ST-1-185, ST-1-192 \& ST-1-207 - Oregon Iron Works (Ref P- } \\
\text { O5064/1.6.4/25 \& 13088/1.6.3.A/21) }\end{array}$ \\
\hline 32 & P-13088 & 1.6.3.B & 19 & $\begin{array}{l}\text { Factory Acceptance Test Procedures (FAT) on Tube Sleeves - OIW Shipment 462-27 - } \\
\text { ST-1-019, ST-1-131, ST-1-157, ST-1-178 \& ST-1-203 - Oregon Iron Works (Ref P. } \\
\text { 05064/1.6.4/26 \& 13088/1.6.3.A/22) }\end{array}$ \\
\hline
\end{tabular}




\begin{tabular}{|c|c|c|c|c|}
\hline & Section & Para & $\begin{array}{l}\text { Submittal } \\
\text { No }\end{array}$ & OESCRIPIION \\
\hline 33 & P-13088 & 1.6.3.B & 20 & $\begin{array}{l}\text { Factory Acceptance Test Procedures (FAT) on Tube Sleeves - OIW Shipment } 462-31 \text { - } \\
\text { ST-1-130, ST-1-190, ST-1-205, ST-1-208 \& ST-1-213 - Oregon Iron Works (Ref P- } \\
\text { O5064/1.6.4/27 \& 13088/1.6.3.A23) }\end{array}$ \\
\hline 34 & P-13088 & 1.6.3.B & 21 & $\begin{array}{l}\text { Factory Acceptance Test Procedures (FAT) on Tube Sleeves - OIW Shipment 462-33 - } \\
\text { ST-1-093, ST-1-133, ST-1-191, ST-1-193 \& ST-1-195 - Oregon Iron Works (Ref P- } \\
\text { 05064/1.6.4/28 \& 13088/1.6.3.A24) }\end{array}$ \\
\hline 35 & $\mathrm{P}-13088$ & 1.6.3.B & 22 & $\begin{array}{l}\text { Factory Acceptance Test Procedures (FAT) on Tube Sleeves - OIW Shipment 462-34 - } \\
\text { ST-1-073, ST-1-123, ST-1-158, ST-1-176 \& ST-1-197 - Oregon Iron Works (Ref P. } \\
\text { 05064/1.6.4/29 \& 13088/1.6.3.A/25) }\end{array}$ \\
\hline 36 & P-13088 & 1.6.3.B & 23 & $\begin{array}{l}\text { Factory Acceptance Test Procedures (FAT) on Tube Sleeves - OIW Shipment } 462-35 \text { - } \\
\text { ST-1-148, ST-1-160, ST-1-169, ST-1-211 \& ST-1-214 - Oregon Iron Works (Ref P- } \\
\text { 05064/1.6.4/30 \& 13088/1.6.3.A/26) }\end{array}$ \\
\hline 37 & $P-13088$ & 1.6.3.B & 24 & $\begin{array}{l}\text { Factory Acceptance Test Procedures (FAT) on Tube Sleeves - OIW Shipment 462-36 - } \\
\text { ST-1-114, ST-1-146, ST-1-163, ST-1-206 \& ST-1-216 - Oregon Iron Works (Ref P- } \\
\text { 05064/1.6.4/31 \& 13088/1.6.3.A/27) }\end{array}$ \\
\hline 38 & $P-13088$ & 1.6.3.B & 25 & $\begin{array}{l}\text { Factory Acceptance Test Procedures (FAT) on Tube Sleeves - OlW Shipment 462-20- } \\
\text { ST-1-014, ST-1-020, ST-1-021, ST-1-122 \& ST-1-136 - Oregon Iron Works (Ref P- } \\
\text { 05064/1.6.4/32 \& 13088/1.6.3.A/28) }\end{array}$ \\
\hline 39 & P-13088 & 1.6.3.B & 26 & $\begin{array}{l}\text { Factory Acceptance Test Procedures (FAT) on Tube Sleeves - OIW Shipment 462-24 - } \\
\text { ST-1-045, ST-1-153, ST-1-165, ST-1-181 \& ST-1-218 - Oregon Iron Works (Ref P- } \\
\text { 06064/1.6.4/33 \& 13088/1.6.3.A29) }\end{array}$ \\
\hline 40 & $\mathrm{P}-13088$ & 1.6.3.B & 27 & $\begin{array}{l}\text { Factory Acceptance Test Procedures (FAT) on Tube Sleeves - OIW Shipment 462-25 - } \\
\text { ST-1-161, ST-1-162, ST-1-170, ST-1-172 \& ST-1-183 - Oregon Iron Works (Ref P- } \\
\text { 05064/1.6.4/34 \& 13088/1.6.3.A/30) }\end{array}$ \\
\hline 41 & P-13088 & 1.6.3.B & 28 & $\begin{array}{l}\text { Factory Acceptance Test Procedures (FAT) on Tube Sleeves - OIW Shipment 462-26 - } \\
\text { ST-1-031, ST-1-159, ST-1-177, ST-1-179 \& ST-1-187 - Oregon lron Works (Ref P- } \\
\text { 05064/1.6.4/35 \& 13088/1.6.3.A/31) }\end{array}$ \\
\hline 42 & P-13088 & 1.6.3.B & 29 & $\begin{array}{l}\text { Factory Acceptance Test Procedures (FAT) on Tube Sleeves - OlW Shipment 462-37 - } \\
\text { ST-1-082, ST-1-175, ST-1-182, ST-1-201 \& ST-1-204 - Oregon Iron Works (Ref P- } \\
\text { 05064/1.6.4/36 \& 13088/1.6.3.A/32) }\end{array}$ \\
\hline 43 & $P-13088$ & 1.6.3.B & 30 & $\begin{array}{l}\text { Factory Acceptance Test Procedures (FAT) on Tube Sleeves - OIW Shipment 462-38 - } \\
\text { ST-1-072, ST-1-085, ST-1-100, ST-1-152 \& ST-1-196 - Oregon Iron Works (Ref P- } \\
\text { 05064/1.6.4/37 \& 13088/1.6.3.A/33) }\end{array}$ \\
\hline 44 & $P-13088$ & 1.6.3.B & 31 & $\begin{array}{l}\text { Factory Acceptance Test Procedures (FAT) on Tube Sleeves - OIW Shipment 462-39- } \\
\text { ST-1-047, ST-1-050, ST-1-106, ST-1-166 \& ST-1-220 - Oregon Iron Works (Ref P- } \\
\text { 05064/1.6.4/38 \& 13088/1.6.3.A/34) }\end{array}$ \\
\hline 45 & $P-13088$ & 1.6.3.B & 32 & $\begin{array}{l}\text { Factory Acceptance Test Procedures (FAT) on Tube Sleeves - OIW Shipment } 462-40 \text { - } \\
\text { ST-1-054, ST-1-058, ST-1-067, ST-1-094 \& ST-1-177 - Oregon Iron Works (Ref P- } \\
\text { 05064/1.6.4/39 \& 13088/1.6.3.A/35) }\end{array}$ \\
\hline 46 & $\mathrm{P}-13088$ & 1.6.3.B & 33 & $\begin{array}{l}\text { Factory Acceptance Test Procedures (FAT) on Tube Sleeves - OIW Shipment 462-46 - } \\
\text { ST-1-029, ST-1-066, ST-1-101 \& ST-1-150 - Oregon Iron Works (Ref P-05064/1.6.4/40 } \\
\text { \& 13088/1.6.3.A/37) }\end{array}$ \\
\hline
\end{tabular}




\begin{tabular}{|c|c|c|c|c|}
\hline & Section & Para & Submittal & DESORIPION \\
\hline 47 & P-13088 & 1.6.3.B & 34 & $\begin{array}{l}\text { Factory Acceptance Test Procedures (FAT) on Tube Sleeves - OIW Shipment } 462-44 \text { - } \\
\text { ST-1-034, ST-1-042, ST-1-043, ST-1-065 \& ST-1-098 - Oregon Iron Works (Ref P- } \\
\text { 05064/1.6.4/41 \& 13088/1.6.3.A/38) }\end{array}$ \\
\hline 48 & P-13088 & 1.6.3.B & 35 & $\begin{array}{l}\text { Factory Acceptance Test Procedures (FAT) on Tube Sleeves - OIW Shipment } 462-15 \text { - } \\
\text { ST-1-030, ST-1-037, ST-1-046, ST-1-092 \& ST-1-180 - Oregon Iron Works (Ref P. } \\
\text { 05064/1.6.4/42 \& 13088/1.6.3.A/39) }\end{array}$ \\
\hline 49 & $P-13088$ & 1.6.3.B & 36 & $\begin{array}{l}\text { Factory Acceptance Test Procedures (FAT) on Tube Sleeves - OIW Shipment 462-43- } \\
\text { ST-1-033, ST-1-049, ST-1-059, ST-1-068 \& ST-1-090 - Oregon Iron Works (Ref P- } \\
\text { 05064/1,6.4/43 \& 13088/1.6.3.A/40) }\end{array}$ \\
\hline 50 & $\mathrm{P}-13088$ & $1.6 .3 . \mathrm{B}$ & 37 & $\begin{array}{l}\text { Factory Acceptance Test Procedures (FAT) on Tube Sleeves - OIW Shipment } 462-45 \text { - } \\
\text { ST-1-039, ST-1-089, ST-1-102, ST-1-109 \& ST-1-186 - Oregon Iron Works (Ref P- } \\
\text { 05064/1.6.4/44 \& 13088/1.6.3.A/41) }\end{array}$ \\
\hline 51 & $\mathrm{P}-13088$ & 1.6.3.B & 38 & $\begin{array}{l}\text { Factory Acceptance Test Procedures (FAT) on Tube Sleeves - OIW Shipment } 462-42 \text { - } \\
\text { ST-1-007, ST-1-016, ST-1-078, ST-1-113 \& ST-1-188 - Oregon Iron Works (Ref P- } \\
\text { 05064/1.6.4/45 \& 13088/1.6.3.A/42) }\end{array}$ \\
\hline 52 & P-13088 & 1.6.3.B & 39 & $\begin{array}{l}\text { Factory Acceptance Test Procedures (FAT) on Tube Sleeves - OIW Shipment 462-47 - } \\
\text { ST-1-035, ST-1-070, ST-1-086 \& ST-1-199 - Oregon Iron Works (Ref P-05064/1.6.4/46 } \\
\text { \& 13088/1.6.3.A/43) }\end{array}$ \\
\hline 53 & $P-13088$ & 1.6.3.B & 40 & $\begin{array}{l}\text { Factory Acceptance Test Procedures (FAT) on Tube Sleeves - OIW Shipment 462-32 - } \\
\text { ST-1-154, ST-1-155, ST-1-156, ST-1-200 \& ST-1-210 - Oregon Iron Works (Ref P- } \\
\text { 05064/1.6.4/47 \& 13088/1.6.3.A/44) }\end{array}$ \\
\hline 54 & $\mathrm{P}-13088$ & 1.6.3.B & 41 & $\begin{array}{l}\text { Factory Acceptance Test Procedures (FAT) on Tube Sleeves - OIW Shipment } 462-41 \text { - } \\
\text { ST-1-052, ST-1-099, ST-1-116, ST-1-119 \& ST-1-147 - Oregon Iron Works (Ref P. } \\
\text { 05064/1.6.4/48 \& 13088/1.6.3.A45) }\end{array}$ \\
\hline 65 & $P-13088$ & 1.6.3.B & 42 & $\begin{array}{l}\text { Factory Acceptance Test Procedures (FAT) on Tube Sleeves - OIW Shipment 462-28 - } \\
\text { ST-1-194, ST-1-198, ST-1-202, ST-1-209 \& ST-1-215 - Oregon Iron Works (Ref P- } \\
\text { 05064/1.6.4/49 \& 13088/1.6.3.A/46) }\end{array}$ \\
\hline 56 & P-13088 & 1.6.3.B & 43 & $\begin{array}{l}\text { Factory Acceptance Test Procedure (FAT) on Tube Sleeves - ST-1-036 - Oregon Iron } \\
\text { Works (Ref P-05064/1.6.4/50 \& 13088/1.6.3.A47) }\end{array}$ \\
\hline & C-13096 & & & CANISTER/OVERPACK - FLOOR PLUG EMBEDS \\
\hline 57 & C-13096 & 1.6.2.C & 0 & Factory Acceptance Test Procedure (FAT) - PP-009 - Oregon Iron Works \\
\hline 68 & C-13096 & 1.6.2.C & $\mathrm{OA}$ & Factory Acceptance Test Procedure (FAT) - PP-009, Rev 1 - Oregon Iron Works \\
\hline 59 & C-13096 & 1.6.2.C & $\mathrm{OB}$ & Factory Acceptance Test Procedure (FAT) - PP-009, Rev 2 - Oregon Iron Works \\
\hline 60 & C-13096 & 1.6.3.B & 0 & $\begin{array}{l}\text { Report Confirming the Factory Acceptance Test Procedure (FAT) was Executed and the } \\
\text { Results - Dimensional Report of Tube Gauge - Oregon Iron Works (Ref C. } \\
\text { 13096/1.6.4.1/0) }\end{array}$ \\
\hline
\end{tabular}




\section{SNF-6215 REV 0}

\begin{tabular}{|c|c|c|c|c|}
\hline & Sedton & Para & Submital & DESCRIPHON \\
\hline 61 & C-13096 & 1.6.3.B & $\mathrm{OA}$ & $\begin{array}{l}\text { Report Confirming the Factory Acceptance Test Procedure (FAT) was Executed and the } \\
\text { Results - Dimensional Report of Tube Gauge Dated 7/27/96 - Reports of Stud Inspection } \\
\text { for 1A-117 \& 1A-137 - Oregon Iron Works (Ref C-13096/1.6.4.1/0) }\end{array}$ \\
\hline 62 & C-13096 & 1.6.3.B & 1 & $\begin{array}{l}\text { Report Confirming the Factory Acceptance Test Procedure (FAT) was Executed and the } \\
\text { Results - Dimensional Report of Tube Gauge - Oregon Iron Works (Ref C- } \\
\text { 13096/1.6.4.1/0) }\end{array}$ \\
\hline 63 & C-13096 & 1.6.3.B & 2 & $\begin{array}{l}\text { Report Confirming the Factory Acceptance Test Procedure (FAT) was Executed and the } \\
\text { Results - Dimensional Report of Tube Gauge Dated } 7 / 27 / 96 \text { - Documents for Shipment } 3 \\
\text { - (1A) Standard Embeds - Oregon Iron Works (Ref C-13096/1.6.4.1/0) }\end{array}$ \\
\hline 64 & C-13096 & 1.6.3.B & 3 & $\begin{array}{l}\text { Report Confirming the Factory Acceptance Test Procedure (FAT) was Executed and the } \\
\text { Results - Dimensional Report of Tube Gauge Dated 8/3/96 - Documents for Shipment } 4 \text { - } \\
\text { (1A) Standard Embeds - Oregon Iron Works (Ref C-13096/1.6.4.1/0) }\end{array}$ \\
\hline 65 & C-13096 & 1.6.3.B & 4 & $\begin{array}{l}\text { Report Confirming the Factory Acceptance Test Procedure (FAT) was Executed and the } \\
\text { Results - Dimensional Report of Tube Gauge Dated 8/10/96 - Documents for Shipment } 5 \\
\text { - (1A) Standard Embeds - Oregon Iron Works (Ref C-13096/1.6.4.1/0) }\end{array}$ \\
\hline 66 & C-13096 & 1.6.3.B & 5 & $\begin{array}{l}\text { Report Confirming the Factory Acceptance Test Procedure (FAT) was Executed and the } \\
\text { Results - Dimensional Report of Tube Gauge Dated 8/10/96 - Documents for Shipment } 5 \\
\text { - (1A) Standard Embeds - Oregon Iron Works (Ref C-13096/1.6.4.1/0) }\end{array}$ \\
\hline 67 & C-13096 & 1.6.3.B & 6 & $\begin{array}{l}\text { Report Confirming the Factory Acceptance Test Procedure (FAT) was Executed and the } \\
\text { Results - Dimensional Report of Tube Gauge Dated 8/24/96 - Documents for Shipment } 7 \\
- \text { (1A) Standard Embeds(16)/4A Std Embeds(16) - Oregon Iron Works (Ref C- } \\
\text { 13096/1.6.4.1/0) }\end{array}$ \\
\hline 68 & C-13096 & 1.6.3.B & 7 & $\begin{array}{l}\text { Report Confirming the Factory Acceptance Test Procedure (FAT) was Executed and the } \\
\text { Results - Dimensional Report of Tube Gauge Dated 9/6/96 - Documents for Shipment } 8 \text { - } \\
\text { (4A) Standard Embeds (34) - Oregon Iron Works (Ref C-13096/1.6.4.1/0) }\end{array}$ \\
\hline 69 & C-13096 & 1.6.3.B & 8 & $\begin{array}{l}\text { Report Confirming the Factory Acceptance Test Procedure (FAT) was Executed and the } \\
\text { Results - Dimensional Report of Tube Gauge Dated 9/6/96 - Documents for Shipment } 9 \text { - } \\
\text { (4A) Standard Embeds (20) - Oregon Iron Works (Ref C-13096/1.6.4.1/0) }\end{array}$ \\
\hline 70 & C-13096 & 1.6.3.B & 9 & $\begin{array}{l}\text { Report Confirming the Factory Acceptance Test Procedure (FAT) was Executed and the } \\
\text { Results - Dimensional Report of Tube Gauges - Documents for Shipment } 11 \text { - (4A) } \\
\text { Standard Embeds (34) - Oregon Iron Works (Ref C-13096/1.6.4.1/0) }\end{array}$ \\
\hline 71 & C-13096 & 1.6.3.B & 10 & $\begin{array}{l}\text { Report Confirming the Factory Acceptance Test Procedure (FAT) was Executed and the } \\
\text { Results - Dimensional Report of Tube Gauges - Documents for Shipment } 10-(4 A) \\
\text { Standard Embeds (24) \& (2A) Overpack Embeds (6) - Oregon Iron Works (Ref C-13096/ } \\
\text { 1.6.4.1/0) }\end{array}$ \\
\hline 72 & $\mathrm{C}-13096$ & 1.6.3.B & 11 & $\begin{array}{l}\text { Report Confirming the Factory Acceptance Test Procedure (FAT) was Executed and the } \\
\text { Results - Dimensional Report of Tube Gauges - Documents for Shipment } 12-(4 \mathrm{~A}) \\
\text { Standard Embeds (20) (Ref C-13096/1.6.4.1/0) }\end{array}$ \\
\hline
\end{tabular}




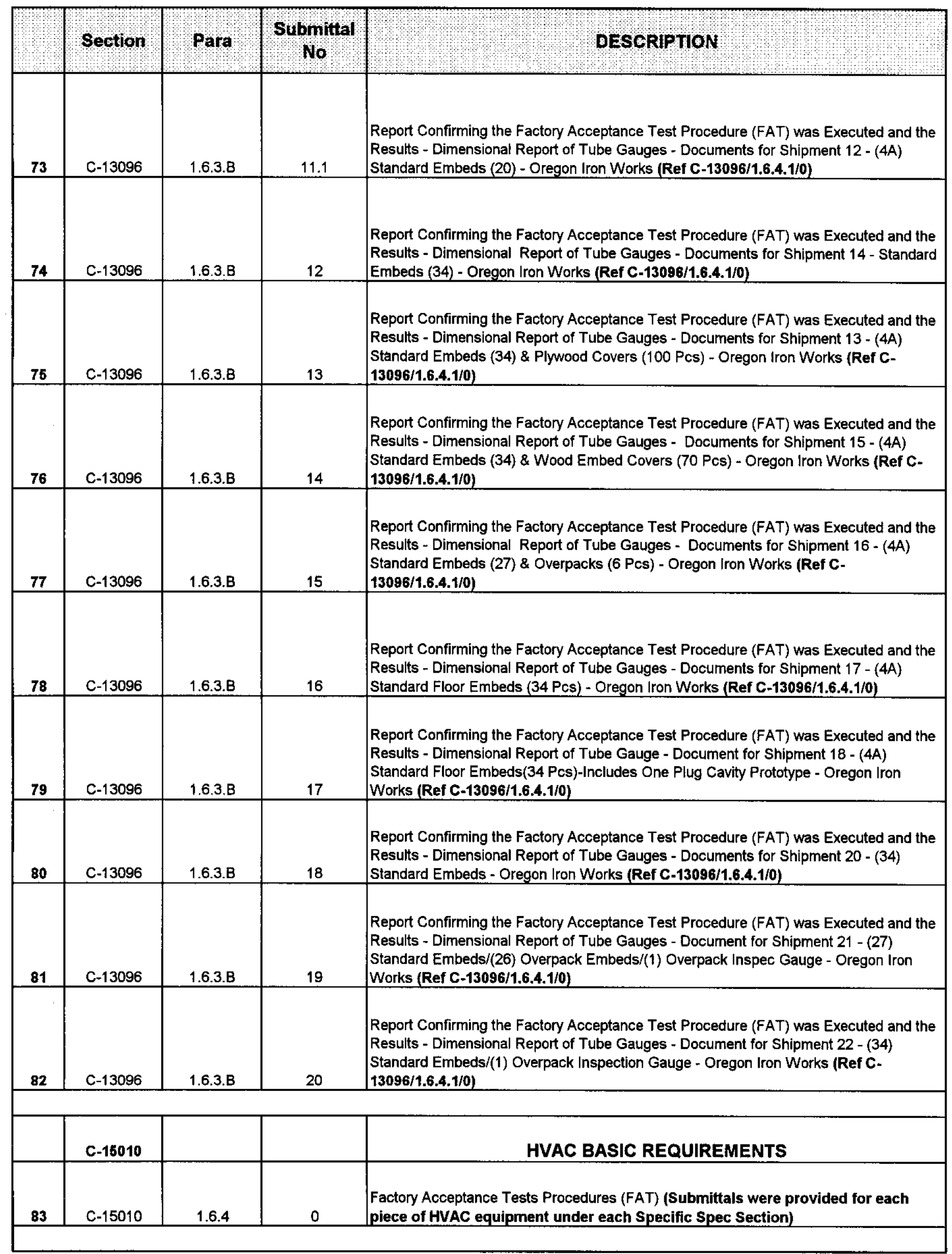




\begin{tabular}{|c|c|c|c|c|}
\hline & Section & Para & Submittal & DESCRIPION \\
\hline & C-16061 & & & $\begin{array}{l}\text { PIPING MATERIAL, FABRICATION, ERECTION AND PRESSURE } \\
\text { TESTING }\end{array}$ \\
\hline \multirow[t]{2}{*}{84} & C-15061 & 1.6.3.A & 0 & Factory Acceptance Test Procedures (FAT) - Thompson Mechanical \\
\hline & C-15138 & & & AIR DIAPHRAGM (SUMP) PUMP \\
\hline \multirow[t]{2}{*}{85} & C-15138 & 1.6 .4 & 0 & $\begin{array}{l}\text { Factory Acceptance Test Procedures (FAT) - Liquid Waste Collection System - } \\
\text { Thompson Mechanical }\end{array}$ \\
\hline & C-15152 & & & INSTRUMENT/PLANT AIR COMPRESSORS \\
\hline 86 & C-15152 & 1.6 .3 & 0 & Certified Factory Acceptance Test Procedures (FAT) - Thompson Mechanical \\
\hline \multirow[t]{2}{*}{87} & C-15152 & 1.6 .3 & 1 & Certified Factory Acceptance Test Procedures (FAT) - Thompson Mechanical \\
\hline & C-15161 & & & VIBRATION ISOLATION DEVICES \\
\hline \multirow[t]{2}{*}{88} & C-15161 & 1.6 .3 & 0 & $\begin{array}{l}\text { Factory Acceptance Test Procedures (FAT) - Thompson Mechanical (No Data } \\
\text { Available) }\end{array}$ \\
\hline & C-15661 & & & AIR COOLED CONDENSING UNITS \\
\hline 89 & C-15661 & 1.6 .5 & 0 & $\begin{array}{l}\text { Factory Acceptance Test Procedures (FAT) - Carrier Model 38AH034-6 CU-001 \& CU- } \\
002 \text { - Thompson Mechanical (Ref Para 2.1.3) }\end{array}$ \\
\hline 90 & C-15661 & 1.6 .5 & OA & $\begin{array}{l}\text { Factory Acceptance Test Procedures (FAT) - Carrier Model 38AH034-6 CU-001 \& CU- } \\
002 \text { - Thompson Mechanical }\end{array}$ \\
\hline & $\mathrm{C}-15680$ & & & AIR-COOLED CHILLER UNIT \\
\hline 91 & $\mathrm{C}-15680$ & 1.6 .5 & 0 & $\begin{array}{l}\text { Factory Acceptance Test Procedure (FAT) - Thompson Mechanical (The Manufacturer } \\
\text { (G\&D Chillers Inc) did not Perform a FAT. The Chiller was Provided as Specified) }\end{array}$ \\
\hline & C-15763 & & & AIR HANDLING UNITS \\
\hline 92 & C-15763 & 1.6 .5 & 0 & $\begin{array}{l}\text { Factory Acceptance Test Procedures (FAT) - Carrier 39TH49 Central Station \& Carrier } \\
\text { 40RMQ008 - Thompson Mechanical (Ref Para 2.1.3) }\end{array}$ \\
\hline 93 & C-15763 & 1.6 .5 & $O A$ & $\begin{array}{l}\text { Factory Acceptance Test Procedures (FAT) - Carrier 39TH49 Central Station \& Carrier } \\
\text { 40RMQ008 - AH-001 \& AH-002 - Thompson Mechanical }\end{array}$ \\
\hline & C-15771 & & & SPLIT SYSTEM HEAT PUMP UNITS \\
\hline 94 & C-15771 & 1.6 .5 & 0 & $\begin{array}{l}\text { Factory Acceptance Test Procedures (FAT) - Carrier 38AQS008 - CU-003 - Thompson } \\
\text { Mechanical (Ref Para 2.1.2.1.9) }\end{array}$ \\
\hline
\end{tabular}




\begin{tabular}{|c|c|c|c|c|}
\hline & Section & Para & Submittal & DESORIPION \\
\hline 95 & C-15771 & 1.6 .5 & $O A$ & $\begin{array}{l}\text { Factory Acceptance Test Procedures (FAT) - Carrier 38AQS008 - AH-003 \& CU-003 - } \\
\text { Thompson Mechanical }\end{array}$ \\
\hline & C-16817 & & & ELECTRIC DUCT HEATERS \\
\hline 96 & C-15817 & 1.6.2.D & 0 & Factory Acceptance Test Procedures (FAT) - EH-001 \& EH-002 - Thompson Mechanical \\
\hline 97 & C-15817 & 1.6 .4 & 0 & $\begin{array}{l}\text { Report Confirming the Factory Acceptance Test Procedures (FAT) was Executed and the } \\
\text { Results - EH-001 \& EH-002 - Thompson Mechanical }\end{array}$ \\
\hline & C-15820 & & & MISCELLANEOUS FANS (HVAC) \\
\hline 98 & C-15820 & 1.6 .4 & 0 & Factory Acceptance Test Procedures (FAT) - EF-001 \& EF-002 - Thompson Mechanical \\
\hline & C-15835 & & & MCO SAMPLING STATION - WELD STATION EXHAUST UNIT \\
\hline 99 & C-15835 & 1.6 .3 & 0 & Factory Acceptance Test Procedures (FAT) - Thompson Mechanical \\
\hline 100 & C-15835 & 1.6 .3 & 1 & Factory Acceptance Test Procedures (FAT) - Thompson Mechanical \\
\hline 101 & C-15835 & 1.6 .6 & 0 & $\begin{array}{l}\text { Report Confirming the Factory Acceptance Test Procedure (FAT) was Executed and the } \\
\text { Results - Thompson Mechanical }\end{array}$ \\
\hline 102 & C-15835 & 1.6 .6 & 1 & $\begin{array}{l}\text { Report Confirming the Factory Acceptance Test Procedure (FAT) was Executed and the } \\
\text { Results - Thompson Mechanical }\end{array}$ \\
\hline & C-16880 & & & INSTRUMENT AIR DRYER SYSTEM \\
\hline 103 & C-15880 & 1.6 .3 & 0 & Certified Factory Acceptance Test Procedures (FAT) - Thompson Mechanical \\
\hline 104 & C-15880 & 1.6 .3 & 1 & Certified Factory Acceptance Test Procedures (FAT) - Thompson Mechanical \\
\hline & C-15881 & & & AIR FILTERS (HVAC) \\
\hline 105 & C-15881 & 1.6 .3 & 0 & $\begin{array}{l}\text { Factory Acceptance Test Procedures (FAT) - Thompson Mechanical (No Data } \\
\text { Available) }\end{array}$ \\
\hline 106 & C-15881 & 1.6 .3 & 1 & Factory Acceptance Test Procedures (FAT) - Thompson Mechanical \\
\hline & C-16950 & & & AIR CONDITIONING INDICATING CONTROLLERS \\
\hline 107 & C-15950 & 1.6 .5 & 0 & Factory Acceptance Test Procedures (FAT) - LP-HV-001 - Power City Electric \\
\hline 108 & C-15950 & 1.6.5 & OA & Factory Acceptance Test Procedures (FAT) - Rev 1 - LP-HV-001 - Power City Electric \\
\hline 109 & C-15950 & 1.6 .5 & 1 & Factory Acceptance Test Procedures (FAT) - Rev 1 - PLC's PLUS - Power City Electric \\
\hline
\end{tabular}




\begin{tabular}{|c|c|c|c|c|}
\hline & Section & Para & Submital & OESCRIPTION \\
\hline 110 & C-15950 & 1.6 .6 & 0 & $\begin{array}{l}\text { Report Confirming the Factory Acceptance Test Procedures (FAT) was Executed and the } \\
\text { Results - LP-HV-001 - Power City Electric (Ref DCN-197) }\end{array}$ \\
\hline 111 & C-15950 & 1.6 .6 & 1 & $\begin{array}{l}\text { Report Confirming the Factory Acceptance Test Procedures (FAT) was Executed and the } \\
\text { Results - PLC's PLUS - Power City Electric }\end{array}$ \\
\hline & C-16122 & & & 15 KV CABLE \\
\hline 112 & $\mathrm{C}-16122$ & 1.6.2.A & 0 & Factory Acceptance Test Procedures (FAT) - Power City Electric \\
\hline 113 & C-16122 & 1.6.2.B & 0 & Factory Production Test Procedures - Power City Electric \\
\hline & C-16150 & & & MOTORS - INDUCTION FOR GENERAL SERVICE \\
\hline 114 & C-16150 & 1.6 .7 & 0 & Factory Acceptance Test Procedures (FATs) - Power City Electric \\
\hline & C-16320 & & & PAD MOUNTED TRANSFORMER \\
\hline 115 & $\mathrm{C}-16320$ & 1.6.4.1 & 0 & Factory Acceptance Test Procedure (FAT) - Power City Electric \\
\hline 116 & C-16320 & 1.6 .5 & 0 & Certified Factory Test Reports - Power City Electric \\
\hline & C-16610 & & & ELECTRICAL REQUIREMENTS FOR PACKAGED EQUIPMENT \\
\hline 117 & C-16610 & 1.6 .8 & 0 & Factory Acceptance Test Procedures (FATs) - Power City Electric \\
\hline & C-17100 & & & DISTRIBUTED CONTROL SYSTEM (DCS) \\
\hline 118 & C-17100 & 1.6 .14 & 0 & Factory Acceptance Test Procedure (FAT) - Power City Electric \\
\hline 119 & C- 17100 & 1.6.14 & 1 & Factory Acceptance Test Procedures (FAT) - PLCs Plus/Power City Electric \\
\hline & C-17601 & & & TEMPERATURE TRANSMITTERS - ELECTONIC \\
\hline 120 & C-17601 & 1.6 .5 & 0 & $\begin{array}{l}\text { Factory Acceptance Test Procedures (FAT) - Thompson Mechanical (Was Not } \\
\text { Performed on TIT-109; Transmitter was Calibrated Onsite \& a CAT Results was } \\
\text { Submitted \& Approved) }\end{array}$ \\
\hline 121 & C-17601 & 1.6 .6 & 0 & $\begin{array}{l}\text { Report Confirming the Factory Acceptance Test Procedures (FAT) was Executed and the } \\
\text { Results - Thompson Mechanical (Was Not Performed on TIT-109; Transmitter was } \\
\text { Calibrated Onsite \& a CAT Results was Submitted \& Approved) }\end{array}$ \\
\hline
\end{tabular}




\begin{tabular}{|c|c|c|c|c|}
\hline & Section & Para & Submittal & DESCRIPIION \\
\hline & C-17612 & & & RESISTANCE TEMPERATURE DETECTORS \\
\hline 122 & $\mathrm{C}-17612$ & 1.6 .5 & 0 & $\begin{array}{l}\text { Factory Acceptance Test Procedures (FAT) - Resistance Temperature Detectors - } \\
\text { Complete Functional Test - Thompson Mechanical }\end{array}$ \\
\hline 123 & C-17612 & 1.6 .6 & 0 & $\begin{array}{l}\text { Report Confirming the Factory Acceptance Test Procedures (FAT) was Executed and the } \\
\text { Results - Resistance Temperature Detectors - Complete Functional Test - Thompson } \\
\text { Mechanical }\end{array}$ \\
\hline & $\mathrm{C}-17614 \mathrm{~B}$ & & & THERMOCOUPLES \\
\hline 124 & $\mathrm{C}-17614 \mathrm{~B}$ & 1.6 .5 & 0 & $\begin{array}{l}\text { Factory Acceptance Test Procedures (FAT) - Thermocouples - Complete Functional Test } \\
\text { - Thompson Mechanical }\end{array}$ \\
\hline 125 & C-17614B & 1.6 .6 & 0 & $\begin{array}{l}\text { Report Confirming the Factory Acceptance Test Procedures (FAT) was Executed and the } \\
\text { Results - Thermocouples - Complete Functional Test - Thompson Mechanical }\end{array}$ \\
\hline & C-17617 & & & PRESSURE TRANSMITTER - ELECTRONIC \\
\hline 126 & C-17617 & 1.6 .5 & 0 & $\begin{array}{l}\text { Factory Acceptance Test Procedures (FAT) - Thompson Mechanical (By Agreement } \\
\text { with FDNW, Calibration of Instruments fulfills the Requirements for the FAT; } \\
\text { Testing was not Available from the Manufacturer; See Attached CAT Results) }\end{array}$ \\
\hline 127 & C-17617 & 1.6 .6 & 0 & $\begin{array}{l}\text { Report Confirming the Factory Acceptance Test Procedures (FAT) was Executed and the } \\
\text { Results - Thompson Mechanical (By Agreement with FDNW, Calibration of } \\
\text { instruments fulfills the Requirements for the FAT; Testing was not Available from } \\
\text { the Manufacturer; See Attached CAT Results) }\end{array}$ \\
\hline & C-17617B & & & PRESSURE TRANSMITTERS - ELECTRONIC \\
\hline 128 & C-17617B & 1.6 .5 & 0 & Factory Acceptance Test Procedures (FAT) - Thompson Mechanical \\
\hline 129 & C-17617B & 1.6.6 & 0 & $\begin{array}{l}\text { Report Confirming the Factory Acceptance Test Procedures (FAT) was Executed and the } \\
\text { Results - Thompson Mechanical }\end{array}$ \\
\hline & C-17621 & & & SELF ACTUATED PRESSURE REGULATORS \\
\hline 130 & C-17621 & 1.6 .7 & 0 & Factory Acceptance Test Procedures (FAT) - Thompson Mechanical \\
\hline 131 & C-17621 & 1.6 .7 & 1 & Factory Acceptance Test Procedures (FAT) - Thompson Mechanical \\
\hline 132 & C-17621 & 1.6 .7 & 2 & Factory Acceptance Test Procedures (FAT) - Thompson Mechanical \\
\hline 133 & C-17621 & 1.6 .8 & 0 & $\begin{array}{l}\text { Report Confirming the Factory Acceptance Test Procedures (FAT) was Executed and the } \\
\text { Results - Thompson Mechanical }\end{array}$ \\
\hline 134 & C-17621 & 1.6 .8 & 1 & $\begin{array}{l}\text { Report Confirming the Factory Acceptance Test Procedures (FAT) was Executed and the } \\
\text { Results - PCV-261 \& PCV-256 - Thompson Mechanical }\end{array}$ \\
\hline
\end{tabular}




\begin{tabular}{|c|c|c|c|c|}
\hline & Section & Para & Submital & DESCRIPIION \\
\hline 135 & C-17621 & 1.6 .8 & 2 & $\begin{array}{l}\text { Report Confirming the Factory Acceptance Test Procedures (FAT) was Executed and the } \\
\text { Results - Thompson Mechanical }\end{array}$ \\
\hline & C.17626 & & & PRESSURE GAUGES \\
\hline 136 & $\mathrm{C}-17626$ & 1.6 .5 & 0 & $\begin{array}{l}\text { Factory Acceptance Test Procedures (FAT) - Thompson Mechanical (No Data } \\
\text { Available) }\end{array}$ \\
\hline 137 & $\mathrm{C}-17626$ & 1.6 .5 & $\mathrm{OA}$ & $\begin{array}{l}\text { Factory Acceptance Test Procedures (FAT) - Thompson Mechanical (Tests were not } \\
\text { Performed by the Manufacturer - Gauges are Factory and Field Calibrated) }\end{array}$ \\
\hline 138 & C-17626 & 1.6 .5 & $\mathrm{OB}$ & $\begin{array}{l}\text { Factory Acceptance Test Procedures (FAT) - Thompson Mechanical (FATs were not } \\
\text { Performed by the Manufacturer but were Factory Calibrated. Field Calibration } \\
\text { Data Sheets were Provided Per Ref: C-17626/1.6.8/\#0) }\end{array}$ \\
\hline 139 & $\mathrm{C}-17626$ & 1.6 .6 & 0 & $\begin{array}{l}\text { Reports Confirming the Factory Acceptance Test Procedures (FAT) was Executed and } \\
\text { the Results - Thompson Mechanical (No Data Available) }\end{array}$ \\
\hline 140 & C-17626 & 1.6 .6 & $\mathrm{OA}$ & $\begin{array}{l}\text { Reports Confirming the Factory Acceptance Test Procedures (FAT) was Executed and } \\
\text { the Results - Thompson Mechanical (Tests were not Performed by the Manufacturer } \\
\text { Gauges are Factory and Field Calibrated) }\end{array}$ \\
\hline 141 & C-17626 & 1.6 .6 & $\mathrm{OB}$ & $\begin{array}{l}\text { Reports Confirming the Factory Acceptance Test Procedures (FAT) was Executed and } \\
\text { the Results - Thompson Mechanical (FATs were not Performed by the Manufacturer } \\
\text { but were Factory Calibrated. Field Calibration Data Sheets were Provided Per Ref: } \\
\text { C-17626/1.6.8/\#0) }\end{array}$ \\
\hline & C-17626B & & & PRESSURE GAUGES \\
\hline 142 & $\mathrm{C}-17626 \mathrm{~B}$ & 1.6 .5 & 0 & $\begin{array}{l}\text { Factory Acceptance Test Procedures (FAT) - Thompson Mechanical (No Data } \\
\text { Available) }\end{array}$ \\
\hline 143 & $\mathrm{C}-17626 \mathrm{~B}$ & 1.6 .5 & $\mathrm{OA}$ & $\begin{array}{l}\text { Factory Acceptance Test Procedures (FAT) - Thompson Mechanical (Tests were not } \\
\text { Performed by the Manufacturer - Gauges are Factory and Field Calibrated) }\end{array}$ \\
\hline 144 & C-17626B & 1.6 .5 & $\mathrm{OB}$ & $\begin{array}{l}\text { Factory Acceptance Test Procedures (FAT) - Thompson Mechanical (FATs were not } \\
\text { Performed by the Manufacturer. Gauges were Factory Calibrated. Field } \\
\text { Calibration Data Sheets were Provided Per Ref: C-17626/1.6.8/\#0) }\end{array}$ \\
\hline 146 & $\mathrm{C}-17626 \mathrm{~B}$ & 1.6 .6 & 0 & $\begin{array}{l}\text { Report Confirming the Factory Acceptance Test Procedures (FAT) was Executed and the } \\
\text { Resuits - Thompson Mechanical (No Data Available) }\end{array}$ \\
\hline 146 & $\mathrm{C}-17626 \mathrm{~B}$ & 1.6 .6 & $\mathrm{OA}$ & $\begin{array}{l}\text { Report Confirming the Factory Acceptance Test Procedures (FAT) was Executed and the } \\
\text { Results - Thompson Mechanical (Tests were not Performed by the Manufacturer - } \\
\text { Gauges are Factory and Field Calibrated) }\end{array}$ \\
\hline
\end{tabular}




\begin{tabular}{|c|c|c|c|c|}
\hline & Section & Para & $\begin{array}{l}\text { Subnittal } \\
\text { No }\end{array}$ & DESCRIPION \\
\hline 147 & $\mathrm{C}-17626 \mathrm{~B}$ & 1.6 .6 & $\mathrm{OB}$ & $\begin{array}{l}\text { Report Confirming the Factory Acceptance Test Procedures (FAT) was Executed and the } \\
\text { Results - Thompson Mechanical (FATs were not Performed by the Manufacturer. } \\
\text { Gauges were Factory Calibrated. Field Calibration Data Sheets were Provided Per } \\
\text { Ref: C-17626/1.6.8/\#0) }\end{array}$ \\
\hline & C-17630 & & & MASS FLOWMETERS \\
\hline 148 & C- 17630 & 1.6 .5 & 0 & Factory Acceptance Test Procedure (FAT) - Thompson Mechanical \\
\hline 149 & $\mathrm{C}-17630$ & 1.6 .5 & 1 & Factory Acceptance Test Procedure (FAT) - Thompson Mechanical \\
\hline 150 & C-17630 & 1.6 .6 & 0 & $\begin{array}{l}\text { Report Confirming the Factory Acceptance Test Procedures (FAT) was Executed and the } \\
\text { Results - Thompson Mechanical }\end{array}$ \\
\hline 161 & $\mathrm{C}-17630$ & 1.6 .6 & 1 & $\begin{array}{l}\text { Report Confirming the Factory Acceptance Test Procedures (FAT) was Executed and the } \\
\text { Results - Thompson Mechanical }\end{array}$ \\
\hline & C-17632 & & & ROTAMETERS \\
\hline 152 & C-17632 & 1.6 .5 & 0 & Factory Acceptance Test Procedures (FAT) - Thompson Mechanical \\
\hline 163 & C-17632 & 1.6 .6 & 0 & $\begin{array}{l}\text { Report Confirming the Factory Acceptance Test Procedures (FAT) was Executed and the } \\
\text { Results - Thompson Mechanical }\end{array}$ \\
\hline & C-17642 & & & ORIFICE PLATES AND RESTRICTION ORIFICES \\
\hline 164 & C-17642 & 1.6 .2 & 0 & $\begin{array}{l}\text { Factory Acceptance Test Procedures (FAT) - Thompson Mechanical (No Data } \\
\text { Available) }\end{array}$ \\
\hline 165 & C-17642 & 1.6 .2 & $\mathrm{OA}$ & $\begin{array}{l}\text { Factory Acceptance Test Procedures (FAT) - Thompson Mechanical (Factory } \\
\text { Acceptance Tests were not Performed on Orifice Plates \& Restriction Orifices) }\end{array}$ \\
\hline 156 & C-17642 & 1.6 .3 & 0 & $\begin{array}{l}\text { Report Confirming Factory Acceptance Test Procedures (FAT) was Executed and the } \\
\text { Results - Thompson Mechanical (No Data Available) }\end{array}$ \\
\hline 157 & $\mathrm{C}-17642$ & 1.6 .3 & $O A$ & $\begin{array}{l}\text { Report Confirming Factory Acceptance Test Procedures (FAT) was Executed and the } \\
\text { Results - Thompson Mechanical (Factory Acceptance Tests were not Performed on } \\
\text { Orifice Plates \& Restriction Orifices) }\end{array}$ \\
\hline & C-17656 & & & GLOBE TYPE CONTROL VALVES \\
\hline 158. & C-17656 & 1.6 .6 & 0 & Factory Acceptance Test Procedures (FAT) - Thompson Mechanical \\
\hline 159 & C-17656 & 1.6 .6 & 1 & Factory Acceptance Test Procedures (FAT) - Thompson Mechanical \\
\hline 160 & $\mathrm{C}-17656$ & 1.6 .7 & 0 & $\begin{array}{l}\text { Report Confirming the Factory Acceptance Test Procedures (FAT) was Executed and the } \\
\text { Results - Globe Type Control Valve (Fisher Control Valve) - Thompson Mechanical }\end{array}$ \\
\hline 161 & C-17656 & 1.6 .7 & 1 & $\begin{array}{l}\text { Report Confirming the Factory Acceptance Test Procedures (FAT) was Executed and the } \\
\text { Results - Thompson Mechanical }\end{array}$ \\
\hline
\end{tabular}




\begin{tabular}{|c|c|c|c|c|}
\hline & Section & Para & Submittal & DESCRIPION \\
\hline & C-17663 & & & PRESSURE RELIEF VALVES \\
\hline 162 & C-17663 & 1.6 .6 & 0 & Factory Acceptance Test Procedures (FAT) - Thompson Mechanical \\
\hline 163 & C-17663 & 1.6 .7 & 0 & $\begin{array}{l}\text { Report Confirming the Factory Acceptance Test Procedures (FAT) was Executed and the } \\
\text { Results - Thompson Mechanical }\end{array}$ \\
\hline & C-17663B & & & PRESSURE RELIEF VALVES \\
\hline 164 & C-17663B & 1.6 .6 & 0 & $\begin{array}{l}\text { Factory Acceptance Test Procedures (FAT) - Thompson Mechanical (No Data } \\
\text { Available) }\end{array}$ \\
\hline 165 & C-17663B & 1.6 .6 & $O A$ & $\begin{array}{l}\text { Factory Acceptance Test Procedures (FAT) - Thompson Mechanical (Valves Provided } \\
\text { as Specified. No Additional Information Available) }\end{array}$ \\
\hline 166 & $\mathrm{C}-17663 \mathrm{~B}$ & 1.6 .7 & 0 & $\begin{array}{l}\text { Report Confirming the Factory Acceptance Test Procedures (FAT) was Executed and the } \\
\text { Results - Thompson Mechanical (No Data Available) }\end{array}$ \\
\hline 167 & C-17663B & 1.6 .7 & $\mathrm{OA}$ & $\begin{array}{l}\text { Report Confirming the Factory Acceptance Test Procedures (FAT) was Executed and the } \\
\text { Results - Thompson Mechanical (Valves Provided as Specified. No Additional } \\
\text { Information Available) }\end{array}$ \\
\hline & C-17674 & & & LEVEL SWITCHES \\
\hline 168 & C-17674 & 1.6 .5 & 0 & Factory Acceptance Test Procedures (FAT) - Thompson Mechanical \\
\hline 169 & C-17674 & 1.6 .6 & 0 & $\begin{array}{l}\text { Report Confirming the Factory Acceptance Test Procedures (FAT) was Executed and the } \\
\text { Results - Thompson Mechanical }\end{array}$ \\
\hline & C-17682 & & & CURRENT TO PNEUMATIC CONVERTERS \\
\hline 170 & C-17682 & 1.6 .5 & 0 & $\begin{array}{l}\text { Factory Acceptance Test Procedures (FAT) - Thompson Mechanical (No Data } \\
\text { Available) }\end{array}$ \\
\hline 171 & $\mathrm{C}-17682$ & 1.6 .5 & $\mathrm{OA}$ & $\begin{array}{l}\text { Factory Acceptance Test Procedures (FAT) - Thompson Mechanical (Manufacturer did } \\
\text { not perform FATs - Instruments have been Calibrated) }\end{array}$ \\
\hline 172 & C-17682 & 1.6 .5 & $\mathrm{OB}$ & $\begin{array}{l}\text { Factory Acceptance Test Procedures (FAT) - Thompson Mechanical (No FATs were } \\
\text { Performed. See Attached Calibration Data Sheets) }\end{array}$ \\
\hline 173 & C-17682 & 1.6 .6 & 0 & $\begin{array}{l}\text { Report Confirming the Factory Acceptance Test Procedures (FAT) was Executed and the } \\
\text { Results - Thompson Mechanical (No Data Available) }\end{array}$ \\
\hline 174 & C-17682 & 1.6 .6 & $\mathrm{OA}$ & $\begin{array}{l}\text { Report Confirming the Factory Acceptance Test Procedures (FAT) was Executed and the } \\
\text { Results - Thompson Mechanical (Manufacturer did not perform FATs - Instruments } \\
\text { have been Calibrated) }\end{array}$ \\
\hline 175 & C- 17682 & 1.6 .6 & $\mathrm{OB}$ & $\begin{array}{l}\text { Report Confirming the Factory Acceptance Test Procedures (FAT) was Executed and the } \\
\text { Results - Thompson Mechanical (No FATs were Performed. See Attached } \\
\text { Calibration Data Sheets) }\end{array}$ \\
\hline
\end{tabular}




\begin{tabular}{|c|c|c|c|c|}
\hline & Section & Para & Submittal & BESCRIPTION \\
\hline & C-17697 & & & SMOKE DETECTORS \\
\hline 176 & C-17697 & 1.6 .5 & 0 & Factory Acceptance Test Procedures (FAT) - Power City Electric \\
\hline 177 & C-17697 & 1.6 .5 & 1 & Factory Acceptance Test Procedures (FAT) - Power City Electric \\
\hline 178 & C-17697 & 1.6 .5 & $1 \mathrm{~A}$ & Factory Acceptance Test Procedures (FAT) - Power City Electric \\
\hline 179 & C-17697 & 1.6 .6 & 0 & $\begin{array}{l}\text { Report Confirming the Factory Acceptance Test Procedures (FAT) was Executed and the } \\
\text { Results - Power City Electric }\end{array}$ \\
\hline & C-17703 & & & INSTRUMENT PIPING MATERIALS \\
\hline 180 & $\mathrm{C}-17703$ & 1.6 .6 & 0 & Factory Acceptance Test Procedures (FAT) - Thompson Mechanical \\
\hline & C. $17703 B$ & & & INSTRUMENT PIPING MATERIALS \\
\hline 181 & C-17703B & 1.6 .6 & 0 & $\begin{array}{l}\text { Factory Acceptance Test Procedures (FAT) - Thompson Mechanical (FAT Procedures } \\
\text { are not Performed on Instrument Tubing \& SwagBlock Fittings) }\end{array}$ \\
\hline & C-17864 & & & $\begin{array}{c}\text { INSTRUMENTS FURNISHED WITH MECHANICAL EQUIPMENT } \\
\text { CAINSTER STORAGE BUILDING }\end{array}$ \\
\hline 182 & C-17864 & 1.6 .11 & 0 & $\begin{array}{l}\text { Factory Acceptance Test Procedures (FAT) - Thompson Mechanical (No Work was } \\
\text { Performed on Vendor - Instruments Provided with their Equipment) }\end{array}$ \\
\hline 183 & C-17864 & 1.6 .12 & 0 & $\begin{array}{l}\text { Report Confirming the Factory Acceptance Test Procedures (FAT) was Executed and the } \\
\text { Results - Thompson Mechanical (No Work was Performed on Vendor - Instruments } \\
\text { Provided with their Equipment) }\end{array}$ \\
\hline & C-17864B & & & $\begin{array}{l}\text { INSTRUMENTS FURNISHED WITH MECHANICAL EQUIPMENT } \\
\text { CAINSTER STORAGE BUILDING }\end{array}$ \\
\hline 184 & C-17864B & 1.6.11 & 0 & $\begin{array}{l}\text { Factory Acceptance Test Procedures (FAT) - Thompson Mechanical (No Work was } \\
\text { Performed on Vendor - Instruments Provided with their Equipment) }\end{array}$ \\
\hline 185 & $\mathrm{C}-17864 \mathrm{~B}$ & 1.6.12 & 0 & $\begin{array}{l}\text { Report Confirming the Factory Acceptance Test Procedures (FAT) was Executed and the } \\
\text { Results - Thompson Mechanical (No Work was Performed on Vendor - Instruments } \\
\text { Provided with their Equipment) }\end{array}$ \\
\hline
\end{tabular}




\section{DISTRIBUTION SHEET}

\begin{tabular}{|c|c|c|c|c|c|}
\hline \multirow{2}{*}{$\begin{array}{l}\text { To } \\
\text { Distribution }\end{array}$} & \multirow{2}{*}{\multicolumn{3}{|c|}{$\begin{array}{l}\text { From } \\
\text { CSB Subproject }\end{array}$}} & \multicolumn{2}{|l|}{ Page 1 of 1} \\
\hline & & & & \multicolumn{2}{|c|}{ Date $4-26-00$} \\
\hline \multirow{2}{*}{\multicolumn{4}{|c|}{$\begin{array}{l}\text { Project Title/Work Order } \\
\text { SNF-62 T } 8, \text { Rev. } 0\end{array}$}} & \multicolumn{2}{|c|}{ EDT No 628690} \\
\hline & & & & \multirow{2}{*}{\multicolumn{2}{|c|}{ ECN No. N/A }} \\
\hline \multicolumn{4}{|c|}{$\begin{array}{l}\text { Spent Nuclear Fuel Project Canister Storage Building Factory Acceptance Test } \\
\text { Procedures }\end{array}$} & & \\
\hline Name & MSIN & $\begin{array}{l}\text { Text } \\
\text { With All } \\
\text { Attach. }\end{array}$ & Text Only & $\begin{array}{l}\text { Attach./ } \\
\text { Appendix } \\
\text { Only }\end{array}$ & $\begin{array}{c}\text { EDT/ECN } \\
\text { Only }\end{array}$ \\
\hline G. D. Bazinet & S8-06 & $\mathrm{X}$ & & & \\
\hline R. J. Jablonski & S1-53 & $X$ & & & \\
\hline S. A. Kreig & S8-06 & $\mathrm{X}$ & & & \\
\hline J. H. Mortimer & S8-06 & $\mathrm{X}$ & & & \\
\hline CSB Project Files & S8-06 & $X$ & & & \\
\hline Start-up Library & B2-64 & $\mathrm{X}$ & & & \\
\hline
\end{tabular}

